Rodrigo Kaufmann Peña ${ }^{\rrbracket}$

\title{
La Reforma Constitucional
}

\section{The constitutional reform}

\begin{abstract}
Resumen
El presente artículo se refiere a los mecanismos que las constituciones establecen para su modificación. Está dividido en dos secciones. En la primera de ellas, se postula que la Constitución es especial por su relación con la política y que sus mecanismos de reforma son una manifestación de dicha relación. A continuación, se plantea que la especialidad de los mecanismos de reforma constitucional responde esencialmente a dos objetivos, que son a su vez consecuencia de dicha relación: en primer lugar, garantizar vías de participación en el proceso de reforma, que excedan de los canales propios de la democracia representativa, y en segundo, generar estabilidad. En la segunda sección, se estudian los mecanismos de reforma que han regido en Chile a la luz de los criterios señalados.
\end{abstract}

\section{Palabras Claves}

Reforma constitucional - participación - estabilidad

"Licenciado en Ciencias Juridicas y Sociales por la Universidad de Chile, Ayudante Adjunto de Derecho Administrativo, correo electrónico: rodrigo. kaufmann@gmail.com.

\begin{abstract}
The article deals with the mechanisms that modern Constitutions establish for its own reform. It is divided into two sections. The first section explores the idea that Constitutions have a special relation to Politics and that the mechanisms for their reform are a consequence of that relationship. These mechanisms pursue two main goals, and both of them can be considered an expression of this Constitutional feature. These two goals are, in the first place, participation, establishing ways of popular intervention that exceed the ones that exist in representative democracy; and secondly, the generation of a certain level of political stability. The second section of this article studies the mechanisms set forth in Chilean Constitutions throughout the country's history, and analyzes them considering the two goals previously identified.
\end{abstract}

\section{Keywords}

Constitutional reform- participation - stability 


\section{La naturaleza de la Constitución y su reforma}

En su obra clásica ${ }^{1}$, James Bryce distinguió entre las constituciones rígidas y las flexibles. Tienen la naturaleza de flexibles las que se encuentran en el mismo nivel jerárquico, provienen de la(s) misma(s) autoridad(es) y son abolidas o modificadas de acuerdo al mismo procedimiento que el resto de las leyes, mientras que las constituciones rígidas se encuentran en un nivel jerárquico superior al de las demás normas jurídicas del respectivo ordenamiento, no son dictadas por la misma autoridad que estas, sino que por una superior o especial, y su reforma o modificación es objeto de un procedimiento especial.

La idea central de este artículo es que el régimen jurídico especial a que está sometida la modificación de una constitución (rígida) es una manifestación de su naturaleza política.

Las relaciones entre el Derecho y la política pueden ser caracterizadas de diversas formas. Dieter Grimm, por ejemplo, concibe la relación de cinco maneras: Derecho como objetivo, producto, herramienta, marco y medida de la política ${ }^{2}$. Sin duda que tal relación puede predicarse de cualquier norma jurídica, y no exclusivamente de la Constitución. Sin embargo, existen también dimensiones en las que las relaciones entre la Constitución y la política presentan una naturaleza tal que se diferencian de la relación que cualquier otra norma jurídica del ordenamiento puede tener con la actividad política. En efecto, la Constitución no es únicamente el resultado o la herramienta, es decir, la materialización de la actividad política, sino que, en un Estado de Derecho, cumple también una función propiamente fundante o constitutiva de la actividad política. En palabras de Dieter Grimm "[l] a constelación a partir de la que se originaron las Constituciones ya no existe. La idea de la soberanía popular se ha fortalecido políticamente de tal forma que ya no se trata de limitar una monarquía existente, sino de la constitución del poder público democrático. La Constitución pierde, así, su carácter contractual (de pacto) y se transforma en una pieza en virtud de la cual la sociedad se organiza a sí misma”3. Es justamente la dimensión de la Constitución como fundamento de la actividad política la que presenta una naturaleza distinta a la relación de las demás normas jurídicas con la actividad política.

Esa relación especial y la función, en cierto sentido, de intermediación constante entre la dimensión política y la jurídica, imponen condiciones especiales a su eficacia. En palabras de Humberto Nogueira, "[u]na contitución solo tendrá durabilidad si es obra de la participación y adhesión de los diversos sectores que integran la sociedad política, y el conjunto de la colectividad la hace suya (...). Lo decisivo continúa siendo aclarar la transición del acto político a la norma jurídica.

BRYCE (1988)

La cita aparece en STERN (1984), p. 23.

GRIMM (1991), p. 318. 
La voluntad, el mandado solo puede convertirse en derecho si es seguido, si es reconocido, si es aceptado ("consentido") como algo que debe tener vigencia" ${ }^{\text {. }}$

Lo anterior parece ser igualmente válido para una reforma constitucional, por cuanto, a pesar de ser el poder de reforma de la Constitución un poder jurídico, juridificado o constituido, al incorporarse nuevas disposiciones a la Carta Fundamental de un ordenamiento jurídico, pasarán a cumplir la función, y a ser evaluadas bajo los mismos criterios de legitimidad que el instrumento del que pasan a formar parte.

Considerando las particularidades de la Constitución, la especial configuración de los mecanismos de reforma constitucional puede ser explicada en base a dos elementos.

Por una parte, el elemento de la legitimidad, que implica que la reforma, y, con ello, la constitución reformada, sean percibidas como manifestación de la voluntad política de la comunidad. De acuerdo a Francisco Zúñiga, "[n]o hay duda que la Constitución es un subsistema normativo iusfundamental válido y eficaz, pero en gran medida se confunde el plano de la eficacia o facticidad con el plano de la legitimidad. La validez de la Constitución es un concepto jurídico, la legitimidad es un concepto político (Linares Quintana)"5. El elemento de la legitimidad se vincula, a este respecto, con las posibilidades de participación de la comunidad a través de mecanismos especiales, distintos de los propios de la democracia representativa tradicional.

Por otra parte, el mecanismo de reforma debe garantizar un nivel mínimo de estabilidad del orden político y permitir su consolidación. Así, debe lograr un difícil equilibro que ya fue señalado por Carrasco Albano, quien indica que "hai que evitar dos escollos en la reforma de las leyes fundamentales de un país, o una facilidad tal para era reforma que produzca la instabilidad en el gobierno, o entorpecimientos que la hagan poco menos que imposible" ${ }^{\text {. }}$

Por consiguiente, el mecanismo de reforma constitucional está determinado por dos elementos: el primero de ellos dice relación con la legitimidad de que debe gozar la reforma (y el texto constitucional reformado). Dicho elemento será tratado a continuación como el elemento de la participación. Por otra parte, viene definido por la necesaria estabilidad que debe garantizar, evitando constantes reformas del sistema político, que impedirían su consolidación. Dicho elemento será denominado el de la estabilidad.

A continuación se estudiarán los mecanismos concretos a través de los cuales se manifiestan los elementos indicados. 


\section{Las manifestaciones de los elementos de participación y estabilidad}

\section{A. La participación}

Como se dijo, una forma de comprender el mecanismo de reforma constitucional, en cuanto mecanismo especial, distinto al mecanismo ordinario de generación o reforma de legislación ordinaria, es entenderlo como una forma de garantizar un grado mayor de participación de la ciudadanía, o de abrir canales especiales, distintos a los propios de la democracia representativa, para que esta se manifieste.

Es posible distinguir diversos medios a través de los cuales dicho objetivo se manifiesta.

\section{El referéndum}

En su versión pura, el referéndum sujeta la reforma de la Constitución a la aprobación de la misma por parte de la mayoría del cuerpo electoral de un determinado país.

En América Latina, la Constitución uruguaya (art. 331) establece la necesidad de ratificación de las reformas por el cuerpo electoral, sea cual fuere el mecanismo concreto de reforma utilizado 7 .

Además, la Constitución peruana (art. 206) establece el referéndum como alternativa, para el caso de no obtenerse acuerdo del Congreso en dos legislaturas ordinarias sucesivas por dos tercios del número legal de congresistas.

También la Constitución de Colombia (arts. 377 y 378) establece mecanismos de referéndum alternativo si la reforma se refiere al Capítulo relativo a los Derechos Fundamentales, sus garantías, los procedimientos de participación popular y el Congreso, debiendo solicitarse por el cinco por ciento de los ciudadanos que integran el censo electoral. También se puede someter a referéndum un proyecto de reforma mediante ley a instancia del Gobierno o de los propios ciudadanos, debiendo la ley ser aprobada por la mayoría de los miembros de ambas Cámaras.

En Europa, en tanto, se contempla un referéndum facultativo en España (art. 167, 3.), a solicitud de la décima parte de los miembros de cualquiera de las Cámaras; en Italia el referéndum es facultativo también (art. 138), debiendo solicitarlo un quinto de los miembros de una Cámara, quinientos mil electores o cinco consejeros regionales.

Francia, por su parte, establece que la reforma únicamente es definitiva con posterioridad a su aprobación por referéndum (art. 89), si bien establece como mecanismo alternativo de aprobación la obtención de un quórum de tres quintos en el Congreso.

\footnotetext{
Uruguay establece cuatro: iniciativa del diez por ciento de los ciudadanos, que se eleva a la Asamblea General; proyecto apoyado por dos quintos del total de componentes de la Asamblea General; proyecto presentados por Senadores, Representantes o el Poder Ejecutivo, aprobados por la mayoría absoluta de la Asamblea General y resuelta por una Convención Nacional Constituyente y por una ley constitucional que requiere de un quórum de dos tercios en cada Cámara.
} 
Además existe el denominado "referéndum indirecto". El mecanismo del referéndum indirecto vincula la aprobación de la reforma a dos legislaturas sucesivas, de manera que la ratificación o aprobación definitiva corresponde a la segunda legislatura, la que ha sido elegida con dicha misión. Así, si bien la ciudadanía no vota sobre la reforma directamente, sí lo hace por un representante, de acuerdo a su intención de voto respecto de la reforma. La revisión de ciertas secciones de la Constitución española (Título preliminar, capítulo sobre derechos y libertades o de la Corona) genera la disolución inmediata de las Cortes, convocándose a una nueva elección de los miembros de las mismas, que deberán ratificar la decisión de revisar las secciones y proceder al estudio del nuevo texto (art. 168, 1.).

\section{Un órgano especial}

La participación de un órgano especial (Asamblea Constituyente o Convención) que garantice mayor representatividad que el cuerpo legislativo ordinario y cuya única función sea la reforma de la Constitución, es otro de los mecanismos especiales que buscan la participación (más) directa de la comunidad política.

La medida se contempla especialmente en Constituciones latinoamericanas. Así, el artículo 331 de la Constitución uruguaya establece, en su letra c), la posibilidad de convocar a una Convención Nacional Constituyente, que se compondrá del doble de miembros que el Poder Legislativo, rigiéndose por el sistema de representación proporcional.

También el artículo 30 de la Constitución argentina establece que la necesidad de reforma será declarada por el Congreso y realizada por una Convención que se convocará al efecto.

Lo propio dispone la Constitución colombiana, que consagra la posibilidad de convocar a una Asamblea Constituyente (art. 376), que es elegida por voto directo de los ciudadanos.

\section{B. La estabilidad}

Por otra parte, y según se ha señalado más arriba, los mecanismos de reforma de la Constitución también deben garantizar una cierta estabilidad; es decir, inmutabilidad o permanencia en el tiempo de los sistemas políticos y de gobierno que consagran. Dicha finalidad se logra a través de distintos mecanismos.

1. Límites materiales

La primera forma de garantizar la estabilidad de disposiciones constitucionales es la de eliminar de plano la posibilidad de su modificación.

Un ejemplo claro de lo anterior lo constituye la Ley Fundamental alemana, que establece en su artículo 79, numeral tercero, que tanto la división de la Federación en Estados federados como la participación de dichos Estados federados en el procedimiento 
legislativo federal y los principios fundamentales contemplados en los artículos 1 y 20 no podrán ser objeto de reformas.

Las Constituciones de Francia e Italia consagran igualmente prohibiciones en cuanto a la afectación de la forma republicana en sus artículos 89 y 139, respectivamente.

El párrafo $4^{\circ}$ del artículo 60 de la Constitución brasileña, en tanto, establece que no serán objeto de deliberación para enmendar la Constitución las materias relacionadas con la forma federal del Estado, el voto secreto, directo, universal y periódico, la separación de los poderes y los derechos y garantías individuales.

2. Mayorías calificadas

Una segunda modalidad es la de exigir mayorías calificadas para la aprobación de la reforma, que impone una exigencia superior a la que debe cumplir una ley ordinaria.

Así, por ejemplo, Francia exige una mayoría de tres quintos (art. 89), que, en caso de alcanzarse, hará innecesario el referéndum

En Alemania se requiere el voto conforme de dos tercios en cada una de las Cámaras, de acuerdo a lo establecido en el artículo 79, numeral 2.

En Latinoamérica, el Perú, en el artículo 206, exige dos tercios, con la dificultad adicional de que dicho quórum debe ser alcanzado en dos legislaturas ordinarias sucesivas.

También México establece un quórum de dos tercios (art. 135) en el Congreso de la Unión, además de exigir la aprobación por la mayoría de las legislaturas de los Estados.

3. Votaciones diferidas

Finalmente, ciertas Constituciones establecen la necesidad de votar dos veces una reforma constitucional, debiendo mediar entre ambas un cierto lapso de tiempo.

Así, la Constitución italiana exige, en el artículo 138, dos deliberaciones sucesivas con un intervalo mínimo de tres meses entre ambas.

La Constitución brasileña, en tanto, establece que cada Cámara del Congreso Nacional, debe votar dos veces la propuesta, debiéndose conseguir en cada caso un quórum de tres quintos (art. 60, \$2).

\section{La reforma constitucional en las Constituciones chilenas}

La historia constitucional chilena muestra una interesante evolución en cuanto a mecanismos de reforma constitucional. Los mecanismos de reforma constitucional ${ }^{8}$ hacen su aparición en la historia institucional chilena con la Constitución de 1818, a partir de la cual doce mecanismos han estado vigentes en nuestro país.

Tanto el Reglamento Constitucional provisorio de 1812 (art. 26) como la Constitución de 1823 (art. 275) establecen cláusulas de intangibilidad. No se han considerado, en consecuencia, entre los mecanismos de reforma. 


\section{A. La Constitución de 1818}

La primera Constitución en establecer una disposición sobre su propia modificación fue el Proyecto de Constitución provisoria de 1818, que en el artículo 5 del párrafo correspondiente a las atribuciones del Senado estableció la siguiente regla:

Art. 5.o. Estará autorizado el Senado para limitar, añadir y enmendar esta Constitución provisoria, según lo exijan las circunstancias.

Como se observa, la regla únicamente radicaba en el Senado la función de reformar la Constitución, sin determinar los trámites que debían cumplirse para dichos efectos. No existía, pues, órgano especial alguno o mecanismos que permitieran la participación de la comunidad política.

\section{B. La Constitución de 1822}

La Carta Fundamental de 1822, en tanto, dispuso en el artículo 246:

Art. 246. Las leyes fundamentales de esta Constitución no podrán variarse sin expresa orden de los pueblos, manifestada solemnemente a sus representantes.

Respecto de la disposición de la Constitución de 1818, se aprecia que en este caso la iniciativa de reforma constitucional se radica en "los pueblos" y no ya en un órgano constituido, si bien dicha intención debe ser comunicada a los representantes. Tampoco se fija, en todo caso, el procedimiento que debe seguirse por los representantes para dichos efectos.

\section{La Constitución de 1828}

La primera Constitución chilena en contemplar en forma expresa un sistema de reforma constitucional fue la de 1828, cuyo artículo 133 disponía:

Artículo 133. El año de 1836 se convocará por el Congreso una gran Convención, con el único y exclusivo objeto de reformar o adicionar esta Constitución, la cual se disolverá inmediatamente que lo haya desempeñado. Una ley particular determinará el modo de proceder, número de que se componga, y demás circunstancia.

Este mecanismo presenta características interesantes. En primer lugar, entrega la modificación de la Constitución a un órgano especial. Se trata del único caso en Chile en que la reforma de la Constitución queda radicada en un órgano distinto al Congreso, potenciando la participación de la comunidad política en el proceso de reforma.

No existen, por otra parte, elementos que busquen la estabilidad de las disposiciones constitucionales; por el contrario, de acuerdo a la propia disposición constitucional, la Carta Fundamental debía ser objeto de revisión ocho años después de su entrada en vigencia. En definitiva, la primacía de la participación por sobre la estabilidad es evidente. 


\section{Constitución de 1833}

La Constitución de 1833 dedica cuatro artículos a la materia de la reforma constitucional.

Artículo 165. Ninguna moción para reforma de uno o más articulos de esta Constitución, podrá admitirse sin que sea apoyada a lo menos por la cuarta parte de los miembros presentes de la Cámara en que se proponga.

Artículo 166. Admitida la moción a discusión, deliberará la Cámara si exijen o no reforma el artículo o artículos en cuestión.

Artículo 167. Si ambas cámaras resolvieren por las dos tercias partes de sufragios en cada una, que el artículo o articulos propuestos exijen reforma, pasará esta resolución al Presidente de la República para los efectos de los artículos 43, 44, 45, 46 i 47.

Artículo 168. Establecida por la lei la necesidad de la reforma, se aguardará la prósima renovación de la Cámara de Diputados, i en la primera sesión que tenga el Congreso, después de esta renovación, se discutirá i deliberará sobre la reforma que haya de hacerse, debiendo tener origen la lei en el Senado, conforme a lo prevenido en el artículo 40; i procediéndose según lo dispone la Constitución para la formación de las demás leyes.

Con respecto al mecanismo contemplado en su antecesora directa, varias modificaciones llaman la atención.

En primer lugar, el proceso de reforma constitucional queda entregado a los Poderes Legislativo y Ejecutivo, situación que se mantiene, en términos generales, hasta el día de hoy. La potestad de reforma constitucional no recae, en consecuencia, en un órgano distinto al que ejerce la potestad legislativa ordinaria, como ocurría en el caso de la Constitución de 1828. Sin embargo, lo anterior no implica que el mecanismo de reforma no contemple un mecanismo institucional especial de participación; en efecto, el artículo 168 consagra el denominado referéndum indirecto.

Notable es el cambio con respecto a la Constitución de 1828 en cuanto a la estabilidad de sus disposiciones, pues no solo no establece limitaciones temporales a la vigencia del texto constitucional, sino que establece un procedimiento previo, "habilitante", para la discusión de la reforma constitucional propiamente tal. Así, de acuerdo a lo dispuesto en el artículo 165, la moción para modificar uno de los artículos de la Constitución debe cumplir una primera exigencia: ser apoyada por la cuarta parte ${ }^{9}$ de los miembros de la Cámara en la que la moción se origine.

En segundo lugar, para proceder a la reforma de uno o más artículos de la Constitución, debía declararse previamente que dicho(s) artículo(s) "exigía(n)" reforma, cuestión que debía realizarse por ley. Pero ni siquiera la declaración para declarar reformable una

\footnotetext{
El carácter de limitación de la disposición queda de manifiesto al comparar la regla señalada con la aplicable para la presentación de mociones ordinarias: al efecto, la primera parte del artículo 40 de la Constitución de 1833 disponía que "[l]as leyes pueden tener principios en el Senado o en la Cámara de Diputados a proposición de uno de sus miembros, o por mensaje que dirija el Presidente de la República.".
} 
o más disposiciones constitucionales quedaba sometida a las reglas ordinarias de producción legislativa; la "exigencia de reforma" de una disposición constitucional debía contar con el voto conforme de las dos terceras partes en cada una de las Cámaras, e incluso con posterioridad a dicho trámite y habiéndose conseguido los quórums señalados podía el Presidente de la República desechar el proyecto en su totalidad, de acuerdo a lo dispuesto en el artículo 45 de la Constitución de 1833, al que hace expresa referencia el artículo 167.

La modificación de la Constitución se realizaba con posterioridad a la dictación de la ley que declaraba reformable uno o más artículos de la Constitución e inmediatamente después de la siguiente renovación del Congreso: la reforma se tramitaba de acuerdo a las reglas de la formación de leyes ordinarias por el nuevo Congreso, con la salvedad de tener que originarse en el Senado.

En consecuencia, la necesidad de estabilización se manifiesta en forma clara a partir de los trámites a los que se ha hecho referencia, mientras que la participación especial se garantizaba a través del mecanismo del referéndum indirecto.

\section{Reforma}

El mecanismo original de reforma de la Constitución de 1833 fue modificado por la Ley de 15 de enero de 1882, quedando con la siguiente redacción:

Artículo 165. La reforma de las disposiciones constitucionales podrá proponerse en cualquiera de las Cámaras, en conformidad a lo dispuesto en la primera parte del artículo 40.

No podrá votarse el proyecto de reforma en ninguna de las Cámaras sin la asistencia de la mayoría absoluta de los miembros de que se compone.

Para la aprobación del proyecto de reforma, las Cámaras se sujetarán a las reglas establecidas en los artículos 41 , 49 i 51.

Artículo 166. El proyecto de reforma aprobado por ambas Cámaras que, en conformidad de lo dispuesto en el artículo 43, se pasare al Presidente de la República, sólo podrá ser observado por éste para proponer modificaciones o correcciones a las reformas acordadas por el Congreso.

Si las modificaciones que el Presidente de la República propusiere fueren aprobadas en cada Cámara por la mayoría de los dos tercios de los miembros presentes, en conformidad a lo dispuesto en el inciso $2^{\circ}$ del artículo anterior, se devolverá el proyecto al Presidente de la República en la forma que la ha presentado para su promulgación.

Si las Cámaras aprobaren solo en parte las modificaciones o correcciones hechas por el Presidente de la República i no insistieren por mayoría de los dos tercios en las otras reformas aprobadas por el Congreso i que el Presidente modifica, se tendrán por aprobadas las reformas en que el Presidente de la República i las Cámaras están de acuerdo, i se devolverá el proyecto en esta forma para su promulgación. 
Cuando las Cámaras no aprobaren las modificaciones propuestas por el Presidente de la República e insistieren, por la mayoría de los dos tercios presentes en cada una de ellas, en las reformas ántes aprobadas por el Congreso, se devolverá el proyecto en su forma primitiva al Presidente de la República para que lo promulgue.

Articulo 167. Las reformas aprobadas i publicadas a que se refieren los dos articulos anteriores, se someterán a la ratificación del Congreso que se elija o renueve inmediatamente después de publicado el proyecto de reforma.

Este Congreso se pronunciará sobre la ratificación de las reformas en los mismos términos en que han sido propuestas, sin hacer en ellas alteración alguna.

La deliberación sobre la aceptación i ratificación, principiará en la Cámara en que tuvo orijen el proyecto de reforma, i cada Cámara se pronunciará por la mayoría absoluta del numero de los miembros presentes, que no podrá ser menor que la mayoría absoluta del número de miembros de que cada una se compone.

Ratificado el proyecto de reforma por cada una de las Cámaras, se pasará al Presidente de la República para su promulgación.

Una vez promulgado el proyecto, sus disposiciones formarán parte de esta Constitución i se tendrán por incorporadas en ella.

Las reformas que hubieren de someterse a la ratificación del Congreso inmediato, se publicarán por el Presidente de la República dentro de los seis meses que precedan a la renovación de dicho Congreso, i por lo menos tres meses ántes de la fecha en que hayan de verificarse las elecciones.

Al hacer esta publicación, el Presidente de la República anunciará al país que el Congreso que se va a elegir tiene el encargo de aceptar i ratificar las reformas propuestas.

Cuando el Congreso llamado a ratificar las reformas dejare transcurrir su periodo constitucional sin hacerlo, las reformas se tendrán por no propuestas.

Artículo 168. Convocado el Congreso a sesiones estraordinarias, podrán proponerse, discutirse i votarse en cualquiera de las Cámaras los proyectos de reformas a que se refiere el articulo 165, aun cuando no fueren incluidos en la convocatoria por el Presidente de la República.

El Congreso llamado a deliberar sobre la ratificación de las reformas propuestas, podrá, si asi lo acordaren ambas Cámaras por mayoría absoluta de votos en sesiones que deberán celebrar con la concurrencia también de la mayoría absoluta de los miembros de que se componen, continuar funcionando en sesiones estraordinarias hasta por noventa días, sin necesidad de convocatoria del Presidente de la República para ocuparse esclusivamente de la ratificación.

En todo caso, las Cámaras podrán deliberar sobre la ratificación de las reformas propuestas en las sesiones estraordinarias a que hubieren sido convocadas por el Presidente de la República, aun cuando ese negocio no hubiere sido incluido en la convocatoria.

Como puede apreciarse, la reforma cumplió diversos objetivos. 
En primer lugar, se flexibilizó el trámite de presentación de un proyecto de reforma al eliminarse el quórum mínimo de la cuarta parte de los miembros presentes en la Cámara donde se origina el proyecto. A partir de la modificación, la moción de reforma podía ser presentada por un solo miembro, sea de la Cámara o del Senado. Se facultó también al Presidente de la República para iniciar una reforma constitucional por la vía de un mensaje, lo que le estaba vedado bajo la redacción original de la Constitución de 1833.

La norma de quórum se trasladó de la presentación de la moción a la votación de la misma (asistencia de mayoría absoluta).

En segundo lugar, se eliminó la facultad de veto del Presidente de la República.

Además, se mantuvo el requisito de la participación de dos legislaturas sucesivas. Así, el nuevo artículo 167 establece la necesidad de ratificación de las reformas, fijándose el quórum necesario para dichos efectos (mayoría absoluta de los miembros de cada Cámara). Habiéndose producido la ratificación, el Presidente debía promulgar la reforma, pasando sus disposiciones a formar parte de la Constitución.

La reforma flexibilizó el proceso de reforma, entregando, por otra parte, la posibilidad al Congreso de hacer primar su voluntad sobre la del Presidente de la República mediante el mecanismo de la insistencia. En definitiva, se redujeron las reglas destinadas a la permanencia de las disposiciones constitucionales, manteniendo las relativas a la participación y entregando mayores atribuciones al Congreso en desmedro del Presidente.

\section{E. Constitución de 1925}

La Constitución de 1925 dedicó, en su versión original, tres artículos a establecer el mecanismo para su propia reforma.

Artículo 108. La reforma de las disposiciones constitucionales se someterá a las tramitaciones de un proyecto de lei, salvas las escepciones que a continuación se indican:

El proyecto de reforma necesitará para ser aprobado en cada Cámara, el voto conforme de la mayoría de los Diputados o Senadores en actual ejercicio.

La dos Cámaras, reunidas en sesión pública, con asistencia de la mayoría del total de sus miembros, sesenta días después de aprobado un proyecto en la forma señalada en el inciso anterior, tomarán conocimiento de él y procederán a votarlo, sin mayor debate.

El proyecto que apruebe la mayoría del Congreso Pleno, pasará al Presidente de la República.

Si en el día señalado no se reuniere la mayoría del total de los miembros del Congreso, la sesión se verificará al siguiente, con los Diputados y Senadores que asistan.

Artículo 109. El proyecto solo podrá ser observado por el Presidente de la República, para proponer modificaciones o correcciones a las reformas acordadas por el Congreso Pleno.

Si las modificaciones que el Presidente de la República propusiere, fueren aprobadas por ambas Cámaras, se devolverá el proyecto al Presidente para su promulgación. 
Si las dos Cámaras desecharen todas o algunas de las observaciones del Presidente de la República e insistieren por los dos tercios de sus miembros presentes en la totalidad o parte del proyecto aprobado por ellas, se devolverá al Presidente para su promulgación, o para que, si éste lo estima conveniente, consulte a la Nación, dentro del término de treinta días, los puntos en desacuerdo, por medio de un plebiscito. El proyecto que se apruebe en el plebiscito se promulgará como reforma constitucional.

Artículo 110. Una vez promulgado el proyecto, sus disposiciones formarán parte de la Constitución y se tendrán por incorporadas a ella.

La diferencia más marcada con el régimen de reforma vigente bajo la Constitución de 1833 es la eliminación de la participación de dos legislaturas sucesivas en la aprobación de una reforma constitucional; es decir, del mecanismo del referéndum indirecto, que había sido la vía especial de participación en los procesos de reforma constitucional hasta entonces.

El nuevo procedimiento de reforma establece, en vez del mecanismo de referéndum indirecto que había regido bajo dos modalidades distintas (la del texto original y la posterior a la reforma de 1882), dos trámites separados para la aprobación de la reforma en el Congreso: en primer lugar, la aprobación por cada una de las Cámaras del proyecto por la mayoría absoluta de los miembros que las componen (manteniendo, de esta forma, la regla de quórum existente en el mecanismo reformado del régimen constitucional anterior) y, en segundo lugar, la reunión de ambas Cámaras del Congreso en sesión pública ${ }^{10}$ sesenta días después de la aprobación del proyecto. También se incorpora al proceso de reforma el referéndum o plebiscito, que sin embargo no tiene un carácter propiamente participativo; antes bien se trata de un arbitrio para superar diferencias entre el Congreso Nacional y el Presidente de la República: este último no está ya vinculado por la insistencia del Congreso, sino que puede consultar a la Nación, cuya decisión sí tendrá el carácter de vinculante. Desde la perspectiva del elemento de la participación, es claro que el mecanismo vigente bajo la Constitución anterior garantizaba a la ciudadanía una mayor intervención; los nuevos (la sesión pública y la posibilidad de referéndum) no presentan, en cambio, naturaleza realmente participativa.

En definitiva, una participación directa y vinculante de la ciudadanía se da únicamente en el supuesto de que exista una controversia sobre la reforma constitucional entre los órganos con participación directa en la reforma, esto es, entre el Presidente de la República y el Congreso Nacional.

10 Es interesante notar que la Constitución de 1925 establece dos casos de reunión de ambas Cámaras del Congreso en sesión pública: además de la aprobación de un proyecto de reforma constitucional, dicha reunión también se producía, de acuerdo al artículo 64, cincuenta días después de la votación para elegir al Presidente de la República, con asistencia de la mayoría de sus miembros, y para efectos de proclamar como tal al ciudadano que hubiere obtenido más de la mitad de los sufragios válidamente emitidos. Se trata, como puede apreciarse, de innegable naturaleza política. 
En tanto, desde la perspectiva de la estabilidad se introduce el mecanismo de la votación diferida, debiendo mediar sesenta días entre la aprobación del proyecto de reforma en cada Cámara y la votación del mismo en la sesión pública.

\section{Reforma}

El mecanismo de reforma constitucional establecido por la Constitución de 1925 fue modificado en virtud de la Ley $\mathrm{N}^{\circ} 17.284$, de 23 de enero de $1970^{11}$.

Artículo 108. La reforma de las disposiciones constitucionales se someterá a las tramitaciones de un proyecto de lei, salvas las excepciones que a continuación se indican:

El proyecto de reforma necesitará para ser aprobado en cada Cámara, el voto conforme de la mayoría de los Diputados o Senadores en actual ejercicio.

La dos Cámaras, reunidas en sesión pública, con asistencia de la mayoría del total de sus miembros, sesenta dias después de aprobado un proyecto en la forma señalada en el inciso anterior, tomarán conocimiento de él y procederán a votarlo, sin mayor debate.

El proyecto que apruebe la mayoría del Congreso Pleno, pasará al Presidente de la República.

Si en el día señalado no se reuniere la mayoría del total de los miembros del Congreso, la sesión se verificará al siguiente, con los Diputados y Senadores que asistan.

El proyecto aprobado por el Congreso Pleno no podrá ser rechazado totalmente por el Presidente de la República, quien sólo podrá proponer modificaciones o correcciones, 0 reiterar ideas contenidas en el mensaje o en indicaciones válidamente formuladas por el propio Presidente de la República.

Si las observaciones que formulare el Presidente de la República en conformidad al inciso anterior fueren aprobadas por la mayoría que establece el inciso segundo, se devolverá el proyecto al Presidente para su promulgación.

Artículo 109. El Presidente de la República podrá consultar a los ciudadanos, mediante un plebiscito, cuando un proyecto de reforma constitucional presentado por él sea rechazado totalmente por el Congreso, en cualquier estado de su tramitación. Igual convocatoria podrá efectuar cuando el Congreso haya rechazado total o parcialmente las observaciones que hubiere formulado, sea que el proyecto haya sido iniciado por mensaje o moción.

Sin embargo, esta facultad no podrá ejercerla respecto de reformas constitucionales que tengan por objeto modificar las normas sobre plebiscito prescritas en este artículo.

La convocatoria a plebiscito deberá efectuarse dentro de los treinta dias siguientes a aquél en que una de las Cámaras o el Congreso Pleno deseche el proyecto de reforma o en que el Congreso rechace las observaciones y se ordenará mediante decreto supremo que fijará la fecha de la consulta plebiscitaria, la que no podrá tener lugar antes de treinta dias ni

11 Las modificaciones al texto original de la Constitución de 1925 han sido subrayadas. En adelante, en caso de reformas, se han transcrito únicamente los artículos modificados. 
después de sesenta contados desde la publicación de ese decreto. Transcurrido este plazo sin que se efectúe el plebiscito, se promulgará el proyecto que hubiere aprobado el Congreso.

El decreto de convocatoria contendrá, según corresponda, el proyecto del Presidente de la República rechazado por una de las Cámaras o por el Congreso Pleno, o las cuestiones en desacuerdo que aquél someta a la decisión de la ciudadania. En este último caso, cada una de las cuestiones en desacuerdo deberá ser votada separadamente en la consulta popular.

El Tribunal Calificador de Elecciones comunicará al Presidente de la República el resultado del plebiscito, especificando el texto del proyecto aprobado por la mayoría de los sufragios válidamente emitidos, que deberá ser promulgado como reforma constitucional dentro del plazo que establece el inciso segundo del artículo 55 . La misma comunicación deberá enviar si la ciudadania rechazare las observaciones del Presidente de la República, caso en el cual éste promulgará, en el plazo antes indicado, el proyecto aprobado por el Congreso Pleno.

La ley establecerá normas que garanticen a los partidos politicos que apoyen o rechacen el proyecto o las cuestiones en desacuerdo sometidas al plebiscito, un acceso suficiente a los diferentes medios de publicidad, y dispondrá, en los casos y dentro de los limites que ella señale, la gratuidad de dicha publicidad.

Artículo 110. Una vez promulgado el proyecto, y desde la fecha de su vigencia, sus disposiciones formarán parte de la Constitución y se tendrán por incorporadas a ella.

La reforma introducida tuvo dos efectos. En primer lugar, se limitaron las facultades del Presidente de la República en lo que respecta al rechazo de un proyecto de reforma aprobado por el Congreso, estableciéndose en forma expresa que el rechazo de un proyecto no podía ser total, sin perjuicio de que el texto original de la Constitución de 1925 ya establecía que el veto podía tener por objeto "modificaciones o correcciones".

En segundo lugar, se reguló en forma detallada el procedimiento por el que debía regirse el plebiscito para dirimir diferencias entre el Presidente de la República y el Congreso Nacional en lo relativo a una reforma de la Constitución. Así, se limitó la facultad presidencial de realizar consulta ciudadana a dos hipótesis concretas y se establecieron materias que no podían ser objeto de consulta a la Nación, fijando, además, la época de realización de la consulta plebiscitaria.

\section{F. Constitución de 1980}

La Constitución de 1980 introdujo numerosas reglas especiales, que transformaron el sistema de reforma constitucional en el más complejo y rígido de todos los que han regido en Chile.

Artículo 116. Los proyectos de reforma de la Constitución podrán ser iniciados por mensaje del Presidente de la República o por moción de cualquiera de los miembros del Congreso Nacional, con las limitaciones señaladas en el inciso primero del artículo 62. 
El proyecto de reforma necesitará para ser aprobado en cada Cámara el voto conforme de las tres quintas partes de los diputados y senadores en ejercicio.

Será aplicable a los proyectos de reforma constitucional el sistema de urgencias.

Artículo 117. Las dos Cámaras, reunidas en Congreso Pleno y en sesión pública, con asistencia de la mayoría del total de sus miembros, sesenta días después de aprobado un proyecto en la forma señalada en el artículo anterior, tomarán conocimiento de él y procederán a votarlo sin debate.

Si en el día señalado no se reuniere la mayoría del total de los miembros del Congreso, la sesión se verificará al siguiente con los diputados y senadores que asistan.

El proyecto que apruebe la mayoría del Congreso Pleno pasará al Presidente de la República.

Si el Presidente de la República rechazare totalmente un proyecto de reforma aprobado por el Congreso y éste insistiere en su totalidad por las tres cuartas partes de los miembros en ejercicio de cada Cámara, el Presidente deberá promulgar dicho proyecto, a menos que consulte a la ciudadanía mediante plebiscito.

Si el Presidente observare parcialmente un proyecto de reforma aprobado por el Congreso, las observaciones se entenderán aprobadas con el voto conforme de la mayoría absoluta de los miembros en ejercicio de cada Cámara, y se devolverá al Presidente para su promulgación.

En caso de que las Cámaras no aprueben todas o algunas de las observaciones del Presidente, no habrá reforma constitucional sobre los puntos en discrepancia, a menos que ambas Cámaras insistieren por los dos tercios de sus miembros en ejercicio en la parte del proyecto aprobado por ellas. En este último caso, se devolverá al Presidente la parte del proyecto que haya sido objeto de insistencia para su promulgación, salvo que éste consulte a la ciudadanía para que se pronuncie mediante un plebiscito, respecto de las cuestiones en desacuerdo.

La ley orgánica constitucional relativa al Congreso regulará en lo demás lo concerniente a los vetos de los proyectos de reforma y a su tramitación en el Congreso.

Articulo 118. Las reformas constitucionales que tengan por objeto modificar las normas sobre plebiscito prescritas en el articulo anterior, disminuir las facultades del Presidente de la República, otorgar mayores atribuciones al Congreso o nuevas prerrogativas a los parlamentarios, requerirán, en todo caso, la concurrencia de voluntades del Presidente de la República y de los dos tercios, de los miembros en ejercicio de cada Cámara, y no procederá a su respecto el plebiscito.

Los proyectos de reforma que recaigan sobre los capitulos I, VII, Xy XI de esta Constitución deberán, para ser aprobados, cumplir con los requisitos señalados en el inciso anterior. Sin embargo, el proyecto asi despachado no se promulgará y se guardará hasta la próxima renovación conjunta de las Cámaras, y en la primera sesión que éstas celebren deliberarán y votarán sobre el texto que se hubiera aprobado, sin que pueda ser objeto de modificación alguna. Sólo si la reforma fuere ratificada por los dos tercios de los miembros en ejercicio de 
cada rama del nuevo Congreso se devolverá al Presidente de la República para su promulgación. Con todo, si este último estuviera en desacuerdo, podrá consultar a la ciudadanía para que se pronuncie mediante un plebiscito.

Artículo 119. La convocatoria a plebiscito deberá efectuarse dentro de los treinta días siguientes a aquel en que ambas Cámaras insistan en el proyecto aprobado por ellas, y se ordenará mediante decreto supremo que fijará la fecha de la votación plebiscitaria, la que no podrá tener lugar antes de treinta días ni después de sesenta, contado desde la publicación de dicho decreto. Transcurrido este plazo sin que el Presidente convoque a plebiscito, se promulgará el proyecto que hubiere aprobado el Congreso.

El decreto de convocatoria contendrá, según corresponda, el proyecto aprobado por el Congreso Pleno y vetado totalmente por el Presidente de la República, o las cuestiones del proyecto en las cuales el Congreso haya insistido. En este último caso, cada una de las cuestiones en desacuerdo deberá ser votada separadamente en el plebiscito.

El Tribunal Calificador comunicará al Presidente de la República el resultado del plebiscito, y especificará el texto del proyecto aprobado por la ciudadania, el que deberá ser promulgado como reforma constitucional dentro de los cinco días siguientes a dicha comunicación.

Una vez promulgado el proyecto y desde la fecha de su vigencia, sus disposiciones formarán parte de la Constitución y se tendrán por incorporadas a ésta.

Existen diversos elementos destinados a generar estabilidad de las normas constitucionales.

En primer lugar, el quórum: el proyecto de reforma, que podía ser iniciado por vía de moción o de mensaje, debía ser aprobado por ambas Cámaras con el quórum de tres quintas partes de los diputados y senadores en ejercicio.

También en dicha materia había excepciones: en primer lugar, la reforma de algunas materias quedó sujeta a un quórum de aprobación reforzado: los Capítulos I (Bases de la Institucionalidad), VII (Tribunal Constitucional), X (Fuerzas Armadas, de Orden y Seguridad Pública) y XI (Consejo Nacional de Seguridad) exigían el voto conforme de dos tercios de los miembros en ejercicio de cada Cámara. Además, debía esperarse a la próxima renovación conjunta de las Cámaras para que fueran ratificadas por el mismo quórum de dos tercios, luego de lo cual las reformas pasaban al Presidente para su promulgación, quedando a este, sin embargo, el expediente del plebiscito para consultar acerca de las mismas.

En segundo lugar, quedaban sujetas al mismo quórum de dos tercios las reformas cuyo objeto fueran las normas sobre plebiscito previstas en el artículo 116, disminuir facultades del Presidente o entregar mayores atribuciones al Congreso o a los parlamentarios, casos en los que además, y si bien no se exigía ratificación por parte de una legislatura posterior, sí debía contarse con la concurrencia de voluntad del Presidente de la República. Asimismo, respecto de las materias señaladas no existía la posibilidad de realizar un plebiscito. 
Con posterioridad a su aprobación, el proyecto era ratificado por el Congreso Pleno en sesión pública ${ }^{12}$, a la que debía asistir la mayoría del total de sus miembros, manteniéndose, en consecuencia, otro elemento de estabilidad: la votación diferida.

Luego de dicha aprobación, el proyecto pasaba al Presidente de la República, que podía rechazarlo totalmente (veto) o realizar observaciones, regulándose al efecto el régimen de insistencias y consultas plebiscitarias.

Resulta interesante destacar que la versión original de la Constitución de 1980 exige para la realización de un plebiscito la insistencia por parte del Congreso, el que es, nuevamente, concebido únicamente para efectos de dirimir controversias entre el Presidente y el Congreso Nacional. Por otra parte, no existe, más allá de la sesión pública del Congreso Pleno, un elemento que permita diferenciar el procedimiento de reforma constitucional del procedimiento legislativo ordinario, desde la perspectiva de la legitimidad (política) de la reforma, esto es, el elemento de la participación de la comunidad.

\section{Reformas}

a. El 17 de agosto de 1989 se publicó en el Diario Oficial la Ley $\mathrm{N}^{\circ} 18.825$ de 17 de agosto de 1989, que introdujo modificaciones al sistema de reforma constitucional contemplado en el texto original de la Constitución de 1980.

Artículo 116. Los proyectos de reforma de la Constitución podrán ser iniciados por mensaje del Presidente de la República o por moción de cualquiera de los miembros del Congreso Nacional, con las limitaciones señaladas en el inciso primero del artículo 62.

El proyecto de reforma necesitará para ser aprobado en cada Cámara el voto conforme de las tres quintas partes de los diputados y senadores en ejercicio. Si la reforma recayere sobre los capitulos I, III, VII, X, XI o XIV, necesitará, en cada Cámara, la aprobación de las dos terceras partes de los diputados y senadores en ejercicio.

Será aplicable a los proyectos de reforma constitucional el sistema de urgencias.

Artículo 117. Las dos Cámaras, reunidas en Congreso Pleno y en sesión pública, con asistencia de la mayoría del total de sus miembros, sesenta días después de aprobado un proyecto en la forma señalada en el artículo anterior, tomarán conocimiento de él y procederán a votarlo sin debate.

12 Al igual que en la Constitución de 1925, la otra oportunidad en la que el Congreso Pleno se reúne en sesión pública es con ocasión de la elección del Presidente. Sin embargo, mientras que en la Constitución de 1925, la proclamación correspondía a ambas ramas del Congreso reunidas, bajo el imperio de la Constitución de 1980, dicha proclamación corresponde al Tribunal Calificador de Elecciones (art. 27 de la versión original del texto), correspondiéndole al Congreso Pleno, reunido en sesión pública, tomar conocimiento de la resolución de proclamación. 
Si a la hora señalada no se reuniere la mayoría del total de los miembros del Congreso, la sesión se verificará el mismo día, a una hora posterior que el Presidente del Senado haya fijado en la convocatoria, con los diputados y senadores que asistan.

El proyecto que apruebe la mayoría del Congreso Pleno pasará al Presidente de la República.

Si el Presidente de la República rechazare totalmente un proyecto de reforma aprobado por el Congreso y éste insistiere en su totalidad por las dos terceras partes de los miembros en ejercicio de cada Cámara, el Presidente deberá promulgar dicho proyecto, a menos que consulte a la ciudadanía mediante plebiscito.

Si el Presidente observare parcialmente un proyecto de reforma aprobado por el Congreso, las observaciones se entenderán aprobadas con el voto conforme de las tres quintas o dos terceras partes de los miembros en ejercicio de cada Cámara, según corresponda de acuerdo con el artículo anterior y se devolverá al Presidente para su promulgación.

En caso de que las Cámaras no aprueben todas o algunas de las observaciones del Presidente, no habrá reforma constitucional sobre los puntos en discrepancia, a menos que ambas Cámaras insistieren por los dos tercios de sus miembros en ejercicio en la parte del proyecto aprobado por ellas. En este último caso, se devolverá al Presidente al parte del proyecto que haya sido objeto de insistencia para su promulgación, salvo que éste consulte a la ciudadania para que se pronuncie mediante un plebiscito, respecto de las cuestiones en desacuerdo.

La ley orgánica relativa al Congreso regulará en lo demás lo concerniente a los vetos de los proyectos de reforma y a su tramitación en el Congreso.

Además, la reforma derogó el artículo 118.

Los efectos de la reforma fueron los siguientes: en primer lugar, se modificó el régimen de quórum reforzado para la reforma de la Constitución, añadiendo a los capítulos que requerían del voto conforme de las dos terceras partes de los miembros en ejercicio de cada Cámara, además de los ya contemplados en el texto original, los capítulos III (De los Derechos y Deberes Constitucionales) y XIV (Reforma de la Constitución). La eliminación del artículo 118 implicó además la desaparición del quórum reforzado respecto de proyectos que pretendieran reducir las atribuciones del Presidente o aumentar las del Congreso y la desaparición del mecanismo de referéndum indirecto contemplado en el texto original.

Respecto del rechazo total de un proyecto de reforma por parte del Presidente, el quórum de insistencia fue reducido de tres cuartas a dos terceras de los miembros en ejercicio de cada Cámara, de forma de asimilarlo a uno de los quórum de aprobación ordinaria de (ciertas materias de) la Constitución. De la misma forma, se elevó el quórum de aprobación de observaciones parciales presentadas por el Presidente, que pasó de mayoría absoluta a tres quintas o dos terceras partes de los miembros en ejercicio, dependiendo del quórum necesario para la reforma de una disposición constitucional. 
b. Con posterioridad, la Ley $\mathrm{N}^{\circ} 19.671$ también modificó el régimen de reforma, radicando en el Presidente del Senado la convocatoria al Congreso Pleno establecida en el inciso primero del artículo 117, fijando, además, una nueva época para su celebración.

Artículo 117. Las dos Cámaras, reunidas en Congreso Pleno, serán convocadas por el Presidente del Senado a una sesión pública, que se celebrará no antes de treinta ni después de sesenta días contados desde la aprobación de un proyecto en la forma señalada en el artículo anterior, en la que, con asistencia de la mayoría del total de sus miembros, tomarán conocimiento de él y procederán a votarlo sin debate.

Si a la hora señalada no se reuniere la mayoría del total de los miembros del Congreso, la sesión se verificará el mismo día, a una hora posterior que el Presidente del Senado haya fijado en la convocatoria, con los diputados y senadores que asistan.

El proyecto que apruebe la mayoría del Congreso Pleno pasará al Presidente de la República.

Si el Presidente de la República rechazare totalmente un proyecto de reforma aprobado por el Congreso y éste insistiere en su totalidad por las dos terceras partes de los miembros en ejercicio de cada Cámara, el Presidente deberá promulgar dicho proyecto, a menos que consulte a la ciudadanía mediante plebiscito.

Si el Presidente observare parcialmente un proyecto de reforma aprobado por el Congreso, las observaciones se entenderán aprobadas con el voto conforme de las tres quintas o dos terceras partes de los miembros en ejercicio de cada Cámara, según corresponda de acuerdo con el artículo anterior y se devolverá al Presidente para su promulgación.

En caso de que las Cámaras no aprueben todas o algunas de las observaciones del Presidente, no habrá reforma constitucional sobre los puntos en discrepancia, a menos que ambas Cámaras insistieren por los dos tercios de sus miembros en ejercicio en la parte del proyecto aprobado por ellas. En este último caso, se devolverá al Presidente al parte del proyecto que haya sido objeto de insistencia para su promulgación, salvo que éste consulte a la ciudadanía para que se pronuncie mediante un plebiscito, respecto de las cuestiones en desacuerdo.

La ley orgánica relativa al Congreso regulará en lo demás lo concerniente a los vetos de los proyectos de reforma y a su tramitación en el Congreso.

La reforma entregó un mayor grado de autonomía a la actuación del Congreso, además de reducir el lapso de espera para efectos de la votación diferida, pero sin eliminarla.

c. La reforma constitucional realizada en virtud de la Ley $\mathrm{N}^{\circ} 20.050$ también modificó el mecanismo de reforma de la Constitución.

Articulo 116. Los proyectos de reforma de la Constitución podrán ser iniciados por mensaje del Presidente de la República o por moción de cualquiera de los miembros del Congreso Nacional, con las limitaciones señaladas en el inciso primero del artículo 62.

El proyecto de reforma necesitará para ser aprobado en cada Cámara el voto conforme de las tres quintas partes de los diputados y senadores en ejercicio. Si la reforma recayere sobre 
los capitulos I, III, VII, X, XI o XIV, necesitará, en cada Cámara, la aprobación de las dos terceras partes de los diputados y senadores en ejercicio.

En lo no previsto en este Capitulo, serán aplicables a la tramitación de los proyectos de reforma constitucional las normas sobre formación de la ley, debiendo respetarse siempre los quórums señalados en el inciso anterior.

Artículo 117. El proyecto que aprueben ambas Cámaras pasará al Presidente de la República. Si el Presidente de la República rechazare totalmente un proyecto de reforma aprobado por el Congreso y éste insistiere en su totalidad por la dos terceras partes de los miembros en ejercicio de cada Cámara, el Presidente deberá promulgar dicho proyecto, a menos que consulte a la ciudadanía mediante plebiscito.

Si el Presidente observare parcialmente un proyecto de reforma aprobado por ambas Cámaras, las observaciones se entenderán aprobadas con el voto conforme de las tres quintas o dos terceras partes de los miembros en ejercicio de cada Cámara, según corresponda de acuerdo con el artículo anterior y se devolverá al Presidente para su promulgación.

En caso de que las Cámaras no aprueben todas o algunas de las observaciones del Presidente, no habrá reforma constitucional sobre los puntos en discrepancia, a menos que ambas Cámaras insistieren por los dos tercios de sus miembros en ejercicio en la parte del proyecto aprobado por ellas. En este último caso, se devolverá al Presidente al parte del proyecto que baya sido objeto de insistencia para su promulgación, salvo que éste consulte a la ciudadanía para que se pronuncie mediante un plebiscito, respecto de las cuestiones en desacuerdo.

La ley orgánica relativa al Congreso regulará en lo demás lo concerniente a los vetos de los proyectos de reforma y a su tramitación en el Congreso.

El efecto más llamativo fue la eliminación de la sesión pública, y, en consecuencia, de la votación diferida que el Congreso Pleno realizaba. Con ello, se eliminó el último mecanismo especial de participación, ya puramente simbólico, durante el proceso de reforma constitucional. Lo anterior implica que cualquier forma especial de participación ha desaparecido del mecanismo de reforma, con excepción, desde luego, del plebiscito, que, sin embargo y como se ha señalado, se realizará únicamente en ciertos casos y por iniciativa del Presidente de la República.

d. Finalmente, la última reforma de que ha sido objeto el mecanismo de reforma constitucional se introdujo en virtud de la Ley $\mathrm{N}^{\circ} 20.515$, que modificó las reglas aplicables al plebiscito13.

Artículo 129. La convocatoria a plebiscito deberá efectuarse dentro de los treinta días siguientes aquel en que ambas Cámaras insistan en el proyecto aprobados por ellas, y se ordenará mediante decreto supremo que fijará la fecha de la votación plebiscitaria, la que se celebrará ciento veinte días después de la publicación de dicho decreto si ese día corres-

13 La numeración de los artículos ha cambiado, como consecuencia de la reforma constitucional realizada por la Ley $\mathrm{N}^{\circ} 20.050$. 
pondiente a un domingo. Si asi no fuere, ella se realizará el domingo inmediatamente siguiente. Transcurrido este plazo sin que el Presidente convoque a plebiscito, se promulgará el proyecto que hubiere aprobado el Congreso.

El decreto de convocatoria contendrá, según corresponda, el proyecto aprobado por el Congreso Pleno y vetado totalmente por el Presidente de la República, o las cuestiones del proyecto en las cuales el Congreso haya insistido. En este último caso, cada una de las cuestiones en desacuerdo deberá ser votada separadamente en el plebiscito.

El Tribunal Calificador comunicará al Presidente de la República el resultado del plebiscito, y especificará el texto del proyecto aprobado por la ciudadanía, el que deberá ser promulgado como reforma constitucional dentro de los cinco días siguientes a dicha comunicación.

Una vez promulgado el proyecto y desde la fecha de su vigencia, sus disposiciones formarán parte de la Constitución y se tendrán por incorporadas a ésta.

La modificación se refiere a la época de celebración del plebiscito, sin modificar la estructura del mecanismo.

\section{Conclusiones}

Una forma de explicar que el procedimiento de reforma de una Constitución contemple reglas y trámites distintos a los que rigen para la legislación ordinaria es estimar que dicho procedimiento especial es consecuencia de la naturaleza política de la Constitución. Así, es la relevancia política de esta la que impone a la dictación y también a la modificación de una Constitución exigencias especiales - políticas-, que las reglas y trámites especiales buscan satisfacer. En este trabajo, se ha estimado que las exigencias especiales pueden asociarse a dos elementos fundamentales: la búsqueda de legitimidad política, y la estabilidad del sistema político-institucional.

El estudio de los mecanismos de reforma constitucional que han regido en Chile a la luz de los dos elementos identificados arroja conclusiones interesantes.

Así, en primer lugar, resulta fácil advertir que el elemento de la participación ha perdido progresivamente relevancia en los procedimientos de reforma.

La existencia de un órgano especial en una reforma constitucional tuvo una existencia fugaz en nuestra historia constitucional: solamente la Constitución de 1828 radicó en un órgano distinto al Congreso la atribución de realizar una reforma constitucional.

El mecanismo de referéndum indirecto que contemplaba la Constitución de 1833 fue eliminado de la Constitución de 1925, y si bien se incluyó nuevamente respecto de ciertas materias en el texto original de 1980, la reforma del año 1989 derogó la disposición que lo contenía (inciso segundo del artículo 118). El mecanismo de referéndum directo, en tanto, se contempla en la actualidad únicamente en el caso de controversia sobre el texto de la reforma constitucional entre el Congreso y el Presidente, siendo, 
además, una atribución exclusiva y discrecional de este último. La figura no presenta, por lo tanto, un carácter propiamente participativo, sino que más bien se trata de un arbitrio para solucionar una disputa entre los órganos constitucionales señalados.

Por otra parte, el elemento de la estabilidad muestra el desarrollo contrario: salvo la Constitución de 1828, que fijaba la época de su revisión, todas las Constituciones chilenas han establecido reglas especiales de quórum para su propia modificación, sea de mayoría absoluta o más elevadas como la actualmente vigente, que alcanza las tres quintas partes en general, y dos tercios para determinadas materias.

Así, existen determinadas materias cuya modificación resulta especialmente difícil: entre estas, y junto a las disposiciones relativas a los derechos y deberes constitucionales, objeto de frecuente protección especial, se encuentran las disposiciones sobre el Tribunal Constitucional y las Fuerzas Armadas y de Orden y Seguridad, que no se encuentran comúnmente entre aquellas para cuya modificación se contemplan exigencias de quórum especiales.

También las votaciones diferidas han formado parte de los trámites contemplados para la realización de una reforma constitucional. El lapso de espera entre votaciones fue introducido por el texto original de la Constitución de 1925, manteniéndose vigente hasta la reforma constitucional del año 2005. Como vimos en su oportunidad, la eliminación de la votación diferida implicó, al mismo tiempo, la desaparición del último elemento participativo: la sesión pública.

Doce son los mecanismos de reforma constitucional que han estado vigentes en Chile. Las constantes modificaciones no pueden sorprender: del mecanismo de reforma constitucional vigente depende la estabilidad institucional del país. Más todavía; las disposiciones sobre reforma constitucional son el único mecanismo institucional que permite la actualización de la norma fundamental del ordenamiento jurídico bajo estándares democráticos y en su articulación deben estar presentes mecanismos que aseguren de forma adecuada los dos objetivos que han sido identificados en este trabajo. Como se ha concluido, el mecanismo actualmente vigente en nuestro país parece presentar algunas falencias al respecto, por lo que queda esperar que dicho déficit sea prontamente superado.

\section{Bibliografía}

1. ALONSO AMÉRIGO, JOSÉ Y JEREZ CALDERÓN, JOSÉ JOAQUÍN; El Procedimiento de Revisión Constitucional en dos Legislaturas: un análisis comparado e histórico, en Revista Española de Derecho Constitucional; núm. 76, enero-abril 2006.

2. BRYCE, JAMES; Constituciones flexibles y constituciones rígidas; Centro de Estudios Constitucionales; Madrid; 1988. 
3. CARRASCO ALBANO, MANUEL; Comentarios sobre la Constitución Politica de 1833; Imprenta y Librería del Mercurio; Valparaíso, 1858.

4. GRIMM, DIETER; Die Zukunft der Verfassung; Suhrkamp; Fráncfort del Meno; 1991.

5. NOGUEIRA, HUMBERTO; Consideraciones sobre poder constituyente y reforma de la Constitución en la teoría y la práctica constitucional; en Revista Ius et Praxis; año $15 ; \mathrm{N}^{\circ} 1$.

6. SILVA BASCUNÁAN, ALEJANDRO; Tratado de Derecho Constitucional, La Constitución de 1925; Editorial Jurídica de Chile, Santiago; 1963.

7. STERN, KLAUS; Das Staatsrecht der Bundesrepublik Deutschland,Band I, Grundbegriffe und Grundlagen des Staatsrechts, Strukturprinzipien der Verfassung; 2a Ed.; C.H. Beck; Múnich; 1984.

8. VERDUGO MARINKOVIC, MARIO; Pfeffer Urquiaga, Emilio y Nogueira Alcalá, Humberto; Derecho Constitucional; Editorial Jurídica de Chile, Santiago, 1994.

9. VOLKMANN, UWE; El Derecho Constitucional, entre pretensión normativa y realidad politica, en Revista Teoría y Realidad Constitucional; núm. 21; 2008.

10. ZÚNIGA URBINA, FRANCISCO; "Democracia y Estado de Derecho"; en Zúńiga, F (coordinador), Reforma Constitucional; LexisNexis, Santiago, 2005. 